\title{
Successful Treatment Using Simeprevir, Sofosbuvir and Rituximab of a Severe Form of Hepatitis C Virus-Related Mixed Cryoglobulinemia with Cardiac Involvement
}

\author{
Rafael Mahieu ${ }^{1,2}$, Christian Lavigne², Geoffrey Urbanski², Isabelle Fouchard-Hubert ${ }^{3}$, \\ Alexandra Ducancelle ${ }^{4}$, Françoise Lunel-Fabiani ${ }^{4}$ \\ ${ }^{1}$ Department of Infectious Diseases University Hospital, Angers, France, \\ ${ }^{2}$ Department of Internal Medicine, University Hospital, Angers, France \\ ${ }^{3}$ Department of Hepatology, University Hospital, Angers, France \\ ${ }^{4}$ Department of Biology of Infectious Agents, University Hospital, Angers, France.
}

Received: 29/09/2015

Accepted: 30/09/2015

Published: 11/11/2015

How to cite this article: Mahieu R, Lavigne C, Urbanski G, Fouchard-Hubert I, Ducancelle A, Lunel-Fabiani F. Successful treatment using simeprevir, sofosbuvir and rituximab of a severe form of hepatitis $C$ virus-related mixed cryoglobulinemia with cardiac involvement.

EJCRIM 2015;2:doi:10.12890/2015_000299.

Conflicts of Interests: The authors declare that there are no competing interests.

Acknowledgements: Rafael Mahieu drafted the initial manuscript, and approved the final manuscript as submitted. Christian Lavigne reviewed and revised the manuscript, and approved the final manuscript as submitted. Geoffrey Urbanski revised the manuscript. Isabelle Fouchard-Hubert reviewed and revised the manuscript, and approved the final manuscript as submitted. Alexandra Ducancelle reviewed and revised the manuscript, and approved the final manuscript as submitted. Françoise Lunel-Fabiani reviewed and revised the manuscript, and approved the final manuscript as submitted.

This article is licensed under a Commons Attribution Non-Commercial 4.0 License

\begin{abstract}
Numerous extrahepatic manifestations have been reported in hepatitis C virus (HCV) infection, particularly mixed cryoglobulinemia (MC). $\mathrm{MC}$ generally responds to clearance of HCV under pegylated-interferon plus ribavirin treatment. New direct-acting antiviral agents have been licensed for HCV under different combinations but have not been studied in severe forms of MC. Here, we present a case report describing a life-threatening form of $M C$ with multivisceral involvement, which was successfully treated with concomitant rituximab, sofosbuvir and simeprevir. In light of the rapid clinical remission associated with sustained virological response and the excellent side-effect profile, this treatment should be considered as a first-line therapy in severe forms of MC.
\end{abstract}

\section{LEARNING POINTS}

- A life-threatening form of mixed cryoglobulinemia can occur in hepatitis $C$ virus infection.

- Treatment with concomitant rituximab, sofosbuvir and simeprevir is one therapeutic option with an excellent side-effect profile.

- Despite clinical remission, cryoglobulinemia can remain detectable without clinical manifestations.

\section{KEYWORDS}

Mixed cryoglobulinemia; hepatitis C virus; sofosbuvir; simeprevir 


\section{of Case Reports in \\ Internal Medicine}

\section{INTRODUCTION}

Hepatitis C virus (HCV) infection is major cause of morbidity and mortality, affecting about 160 million people worldwide. Chronic infection is associated with an increased risk of cirrhosis, hepatocellular carcinoma and extrahepatic manifestations. Numerous papers have documented the link between mixed cryoglobulinemia (MC) and HCV infection, which affects 80-90\% of patients with MC. Moreover, cryoglobulins (CGs) are found in 25-50\% of patients with chronic HCV infection ${ }^{[1]}$. MC can be asymptomatic or have a wide range of clinical presentations. In particular, patients may develop symptomatic MC-related small vessel vasculitis with cardiac involvement. Mild forms are commonly cured with antiviral agents only (i.e. combination therapy with interferon and ribavirin), while life-threatening forms may require specific immunosuppressive therapy in addition to antiviral treatment. Recently, first generation direct-acting antiviral agents (DAAs) providing higher sustained virological response (SVR) were approved in Europe. Thus, a new panel of more active DAAs (i.e. sofosbuvir, simeprevir, daclatasvir, ledipasvir) and interferon-free regimens generally consisting of two or three DAAs with or without ribavirin, are now available. Also, a recent study found that long-term clearance of HCV led to persistent resolution or marked improvement of MC, suggesting the need for highly effective antiviral drugs. Here we describe a rare form of severe $\mathrm{MC}$ with cardiac insufficiency, glomerulonephritis and polyneuropathy in a patient with HCV genotype $1 \mathrm{~b}$ infection effectively treated with rituximab and an interferon-free combination of simeprevir and sofosbuvir. To our knowledge, this is the first report of a successful triple therapy for this rare life-threatening complication of HCV-related MC.

\section{CASE PRESENTATION}

A 48-year-old woman was admitted to our hospital for dyspnoea and clinical signs of cardiac insufficiency. She had HCV genotype $1 \mathrm{~b}$ (no previous treatment) with $\mathrm{MC}$ and suffered from psychiatric disorders. Her complaints were progressive shortness of breath over the preceding week, polyarthralgia, chronic pain and loss of feeling in her feet. Tests revealed haemolytic anaemia, acute renal failure, a type II MC and high viral load (Table 1). Clinical examination and transthoracic echocardiography (TTE) were consistent with severe cardiac insufficiency with a left ventricular ejection fraction of $30 \%$. Coronarography and cardiac magnetic resonance imaging did not revealed any other abnormalities. A kidney biopsy showed membranoproliferative glomerulonephritis with subendothelial deposits. A diagnosis of severe $\mathrm{MC}$ with cardiac, renal, neurological, articular and cutaneous involvement was made.The patient was therefore prescribed rituximab $375 \mathrm{mg} / \mathrm{m}^{2}$ weekly for 4 weeks together with a combination of simeprevir and sofosbuvir given for 12 weeks. The patient's weakness and arthralgia improved greatly within a week. No clinical complications were reported. Twelve weeks after the end of treatment, there was no detectable viral load and hepatic and kidney function had greatly improved (Table 1). TTE showed normalization of the left ventricular ejection fraction. After 1 year, the patient was still in clinical remission with SVR although the cryoglobulinemia was still detectable (0.2 g/l).

\begin{tabular}{|c|c|c|c|}
\hline & At baseline & $\begin{array}{c}\text { 1 month after } \\
\text { DAA+rituximab therapy }\end{array}$ & 6-month follow-up \\
\hline Heart failure (NYHA) & Class IV & Class II & Complete Resolution \\
\hline Asthenia & Major weakness & Marked Improvement & Complete Resolution \\
\hline Arthralgia & Intermittent & Stable & Stable \\
\hline AST & $84 \mathrm{IU} / \mathrm{ml}$ & $43 \mathrm{IU} / \mathrm{ml}$ & $20 \mathrm{IU} / \mathrm{ml}$ \\
\hline ALT & $70 \mathrm{IU} / \mathrm{ml}$ & $23 \mathrm{IU} / \mathrm{ml}$ & $10 \mathrm{IU} / \mathrm{ml}$ \\
\hline Creatinine & $169 \mu \mathrm{mol} / \mathrm{I}$ & $110 \mu \mathrm{mol} / \mathrm{l}$ & $75 \mathrm{mmol} / \mathrm{l}$ \\
\hline Proteinuria & $4 \mathrm{~g} / 24 \mathrm{~h}$ & $1.2 \mathrm{~g} / 24 \mathrm{~h}$ & $0.1 \mathrm{~g} / 24 \mathrm{~h}$ \\
\hline FM & 0.1 & & 0.4 \\
\hline FS & $8.3 \mathrm{kPa}$ & & $4.6 \mathrm{kPa}$ \\
\hline Viral Load & $7 \mathrm{log} 10 \mathrm{IU} / \mathrm{ml}$ & Undetectable & Undetectable \\
\hline Cryoglobulinemia & $0.19 \mathrm{~g} / \mathrm{l}$ & $0.19 \mathrm{~g} / \mathrm{l}$ & $0.2 \mathrm{~g} / \mathrm{l}$ \\
\hline C4 & $1 \mathrm{mg} / \mathrm{dl}$ & $2 \mathrm{mg} / \mathrm{dl}$ & $2 \mathrm{mg} / \mathrm{dl}$ \\
\hline RF & Negative & Negative & Negative \\
\hline IgM monoclonal & $412 \mathrm{mg} / \mathrm{dl}$ & $119 \mathrm{mg} / \mathrm{dl}$ & $34 \mathrm{mg} / \mathrm{dl}$ \\
\hline
\end{tabular}

${ }^{1}$ Using the Abbott RealTime HCV assay. AST: alanine aminotransferase; ALT: aspartate aminotransferase; FM: FibroMeter; FS: FibroScan; NYHA: New York Heart Association functional classification; RF: rheumatoid factor. Table 1 Patient data at baseline and during follow-up. 


\section{DISCUSSION}

HCV-related MC may result in acute or progressive life-threatening organ damage with a mortality rate of $20-80 \%{ }^{[2]}$. Much therapeutic progress has been made since the initial treatment of MC with interferon alpha in 1987 and the discovery that HCV was its main aetiological agent. Pegylated interferon-alpha (PEG-IFNa) combined with ribavirin improves overall MC clinical response and SVR in up to 60\% of patients. Rituximab has been successfully used in MC. Several trials have shown that rituximab has better efficacy than conventional treatments ${ }^{[3]}$ and that it seems to be safe in $\mathrm{HCV}$ patients, in contrast to patients with hepatitis B virus. Controlled clinical trials have also demonstrated that PEG-IFN/ribavirin plus rituximab compared to PEG-IFN/ribavirin alone resulted in better clinical remission and CG clearance; those Authors proposed the use of rituximab in patients with severe vasculitis, with or without plasmapheresis, before initiation of antiviral therapy. First-generation DAAs (boceprevir or telaprevir) in combination with PEG-IFNa and ribavirin have been shown to provide higher (up to $75 \%$ ) SVR in HCV genotype 1. They have also been demonstrated to be very effective in $\mathrm{MC}^{[4]}$ but with high rates of side effects. Since 2014, new DAAs with improved side effect profiles and SVR levels (>90\%) have been approved. An interferon-free combination of daily sofosbuvir and simeprevir for 12 weeks was recently studied and validated by international recommendations ${ }^{[5]}$ in patients with genotype $1 \mathrm{HCV}$.

In the absence of other explanations, we attributed the cardiac insufficiency in our patient to MC, despite its rarity in this setting (less than $5 \%$ of $\mathrm{MC}^{[2]}$. Cardiac involvement is associated with a poor survival rate ${ }^{[2]}$. Thus, in light of the association between glomerulonephritis and neuropathy, it was decided to institute a highly effective treatment targeting both autoimmunity and viral triggers. Plasmapheresis was not performed because of patient refusal. The psychiatric disorder contraindicated interferon, while ribavirin was ruled out because of risks associated with the haemolytic anaemia. Finally, rituximab was started after initial management of acute decompensated heart failure, together with daily sofosbuvir $(400 \mathrm{mg}$ ) and simeprevir $(150 \mathrm{mg})$ for 12 weeks. This combination has not been previously studied in severe forms of $M C$, as rituximab is generally started before antiviral treatment. Despite the life-threatening condition of our patient with severe cardiac and kidney involvement, this triple therapy of sofosbuvir, simeprevir and rituximab resulted in a spectacular, rapid and complete clinical recovery with normalization of cardiac function and virological response, without any side effects. This first description of an interferon-free combination of new DAAs with rituximab is particularly encouraging and could be proposed as a first-line treatment for severe HC-related MC.

\section{REFERENCES}

1. Lunel F, Musset L, Cacoub P, Frangeul L, Cresta P, Perrin M, et al. Cryoglobulinemia in chronic liver diseases: role of hepatitis C virus and liver damage. Gastroenterology 1994;106:1291-1300.

2. Terrier B, Karras A, Cluzel P, Collet J-P, Sène D, Saadoun D, et al. Presentation and prognosis of cardiac involvement in hepatitis C virus-related vasculitis. Am J Cardiol 2013;111:265-272.

3. De Vita S, Quartuccio L, Isola M, Mazzaro C, Scaini P, Lenzi M, et al. A randomized controlled trial of rituximab for the treatment of severe cryoglobulinemic vasculitis. Arthritis Rheum 2012;64:843-853.

4. Saadoun D, Resche Rigon M, Pol S, Thibault V, Blanc F, Pialoux G, et al. PegIFNa/ribavirin/protease inhibitor combination in severe hepatitis C virus-associated mixed cryoglobulinemia vasculitis. J Hepatol 2015;62:24-30.

5. American Association for the Study of Liver Diseases. Recommendations for testing, managing, and treating hepatitis C. 2015. www.hcvguidelines.org/full-report-view 